\title{
A Dutch Book against Sleeping Beauties Who Are Evidential Decision Theorists*
}

\author{
Vincent Conitzer \\ Duke University
}

\begin{abstract}
In the context of the Sleeping Beauty problem, it has been argued that so-called "halfers" can avoid Dutch book arguments by adopting evidential decision theory. I introduce a Dutch book for a variant of the Sleeping Beauty problem and argue that evidential decision theorists fall prey to it, whether they are halfers or thirders. The argument crucially requires that an action can provide evidence for what the agent would do not only at other decision points where she has exactly the same information, but also at decision points where she has different but "symmetric" information.
\end{abstract}

Keywords: self-locating beliefs, Sleeping Beauty problem, evidential decision theory, Dutch books.

*This paper appears in Synthese, Volume 192, Issue 9, pp. 2887-2899, October 2015. The final publication is available at Springer via http://dx.doi.org/10.1007/s11229-015-0691-7 


\begin{tabular}{|c|c|c|}
\hline & Heads $(1 / 2)$ & Tails $(1 / 2)$ \\
\hline Monday & awake & awake \\
\hline Tuesday & asleep & awake \\
\hline
\end{tabular}

Figure 1: Coin tosses and corresponding awakenings, as well as their probabilities, in the Sleeping Beauty problem.

\section{Introduction}

The Sleeping Beauty problem (reviewed below) has attracted much attention because it relates to a variety of unresolved philosophical problems 1 It is, in the first place, a puzzle about beliefs. The question of what Beauty should believe upon awakening divides philosophers and others into multiple camps, mainly "halfers" and "thirders" (though finer distinctions can be made). But decision theory has also been pulled into the debate. This is because one natural strategy for adjudicating between the halfer and thirder positions is to evaluate the effects of the candidate beliefs on Beauty's decisions; if one position results in clearly irrational decisions, this would appear to settle the matter. As it turns out, the success of such arguments appears to hinge on which version of decision theory - causal or evidential - Beauty adopts. A natural reaction is to feel disappointment at the fact that a clean resolution to the original question appears elusive. But it is also possible to see opportunity: perhaps variants of the Sleeping Beauty problem actually allow us to adjudicate between causal and evidential decision theory instead. This is the aim of this paper. Of course, this may also help us resolve the original question, insofar as the arguments against halfing or thirding become decisive once the debate between causal and evidential decision theory has been settled in this context.

Let us recall the standard variant of the Sleeping Beauty problem Elga, 2000]. Beauty is put to sleep on Sunday, and a fair coin is tossed. If it lands Heads, she will be briefly awoken on Monday only. If it lands Tails, she will be briefly awoken on Monday, and then again on Tuesday. The table in Figure 1 summarizes this. Whenever she is awoken, she does not remember any previous awakenings, nor does anything in the room indicate to her what day it is. Beauty knows all this throughout. Now let us put ourselves in the shoes of Beauty when she has just been awoken in the experiment. What should be her credence that the coin came up Heads? Halfers believe that the answer is $1 / 2$; after all, the fact that she has been awoken should not tell her anything about the possible (uncentered) world that she is in, because the awakening event is consistent with each of the two possible worlds. In contrast, thirders believe that the answer is $1 / 3$; after all, only $1 / 3$ of the possible awakening events occur in a Heads world, and this would be borne out when repeating the experiment many times. Who is right?

One decision-theoretic approach to answering this question is to attempt to

\footnotetext{
1 Titelbaum 2013] gives a useful overview of these connections.
} 


\begin{tabular}{|r|c|c|}
\hline & Heads $(1 / 2)$ & Tails $(1 / 2)$ \\
\hline Sunday & bet $1:-15$ & bet $1: 15$ \\
\hline Monday & bet $2: 10$ & bet $2:-10$ \\
\hline Tuesday & no bet & bet $2:-10$ \\
\hline total gain from accepting all bets & -5 & -5 \\
\hline
\end{tabular}

Figure 2: The table shows which bet is offered when, as well as the net gain from accepting the bet in the corresponding possible world, for Hitchcock's Dutch book.

construct a Dutch book to which one of the two positions is vulnerable. A Dutch book is a set of bets that the agent in question would all accept individually, but that together ensure that the agent incurs a strict loss overall. In a diachronic Dutch book, the bets are offered at different times; all of the Dutch books discussed in this paper are diachronic. Hitchcock 2004 describes a Dutch book argument against the halfer position. In this Dutch book, the bookie first before Beauty goes to sleep - sells her a bet that costs 15 and pays out 30 if the coin lands Tails 2 Then, each time that Beauty awakens, he sells her a bet that costs 10 and pays out 20 if the coin has landed Heads. The idea is that if Beauty is a halfer, then she will always be willing to accept these bets. (In fact, she will be indifferent between accepting them and not accepting them; it is straightforward to slightly modify the payoffs so that she will strictly prefer to accept them.) Now, if the coin lands Heads, she will buy bet 1 once and bet 2 once, at a total cost of 25 , and bet two will pay out 20 - so she will run a net loss of 5 . On the other hand, if the coin lands Tails, she will buy bet 1 once and bet 2 twice, at a total cost of 35 , and bet 1 will pay out 30 - so again she will run a net loss of 5 . Thus the Dutch book succeeds. The table in Figure 2 summarizes Hitchcock's Dutch book.

However, Draper and Pust 2008] point out that this Dutch book does not succeed against a halfer that accepts evidential decision theory, because, as Arntzenius 2002] pointed out earlier, such an agent would calculate the expected utility of her options differently in the Sleeping Beauty problem. Specifically, consider the situation where Beauty accepts evidential decision theory, has just been awoken, and is now calculating the expected value of accepting bet 2. For this calculation, by EDT, she assumes that, if the coin has come up Tails, she also has accepted or will accept bet 2 on the other day. Thus, in this case, accepting bet 2 (on both days) comes at a cost of 20 , and pays out nothing, for a net loss of 20; whereas in the Heads case, accepting bet 2 (once) comes at a cost of 10 and pays out 20, for a net gain of only 10 . So even if her credence in Heads is as high as $1 / 2$, she will not accept bet 2 , and the Dutch book fails 3

\footnotetext{
${ }^{2}$ To minimize clutter, I will not specify currency units such as dollars or euros.

${ }^{3}$ Draper and Pust do propose a modified Dutch book that involves telling Beauty that it is Monday and then offering a bet; this Dutch book works against some halfers, whether they are causal or evidential decision theorists, but not against so-called "double-halfers" who hold that the correct credence remains $1 / 2$ even after Beauty is told it is Monday.
} 
Briggs 2010] goes further and presents a proof that thirders who accept causal decision theory and halfers who accept evidential decision theory are both immune to Dutch books, and not just in the original Sleeping Beauty problem but also in variants thereof. The intuition for why these two combined positions would be equivalent in this sense is the following. When an evidential decision theorist calculates her utility conditional on accepting a bet (or not accepting it) in a possible world, she assumes that she does the same upon every other awakening, so the effect is multiplied by the number of times she awakens in that possible world. On the other hand, for a thirder, the probability of a possible world is multiplied by the number of times she awakens in that possible world. This ends up having the same effect on the relevant expected utility calculations 4

Briggs' proof implicitly assumes that Beauty, upon an awakening, always has the same information available to her. This rules out variants such as "Technicolor Beauty" [Titelbaum, 2008] where Beauty, upon awakening, sees a piece of paper whose color is not always the same. The Technicolor Beauty variant illustrates that irrelevant additional information, such as the paper's color - which is uncorrelated with the variable of interest - can change the halfer's credence for the variable of interest (at least under a standard interpretation of halfing - but see Footnote 5). Indeed, Briggs does discuss Technicolor Beauty and concludes that this instability of the halfer's credence is a mark against halfing, but this is only after she has completed her discussion of Dutch books. What has not yet been appreciated, to my knowledge, is the following: if Beauty is an evidential decision theorist, then in variants such as Technicolor Beauty where she does not always have the same information available to her upon waking, she is vulnerable to Dutch books, regardless of whether she is a halfer or a thirder. This is what I will demonstrate in what follows. I first introduce a variant of the Sleeping Beauty problem.

\section{The White-Black-Grey (WBG) Sleeping Beauty Variant}

Beauty will be awoken twice, once on Monday and once on Tuesday. As usual, when she is awoken on Tuesday, she has no memory of the previous awakening. The only information available to her upon awakening (besides the information that was available to her at the start of the experiment) is the color of the room in which she is awoken. Two distinct fair coins are tossed to determine the color of the room in which she is awoken on each of the two days. Coin 1 has a white side and a black side; this coin will be used to determine the color of the room in which she is awoken on Monday. Coin 2 has a grey side and a side with the word "opposite" written on it; this coin will be used to determine the color of

\footnotetext{
${ }^{4}$ The idea that thirding goes hand in hand with CDT, and halfing with EDT, also finds support elsewhere, for example in the context of the absentminded driver problem Schwarz, 2014b].
} 


\begin{tabular}{|c|c|c|c|c|}
\hline & WG $(1 / 4)$ & WO $(1 / 4)$ & BO (1/4) & BG (1/4) \\
\hline Monday & white & white & black & black \\
\hline Tuesday & grey & black & white & grey \\
\hline
\end{tabular}

Figure 3: Sequences of coin tosses and corresponding room colors, as well as their probabilities, in the WBG Sleeping Beauty variant.

the room in which she is awoken on Tuesday, where Opposite indicates that the color of the room should be the opposite of what it was on Monday. That is, if the first coin comes up White and the second coin comes up Opposite, then she will be awoken in the black room on Tuesday, and vice versa. The table in Figure 3 shows the resulting possibilities for the sequence of rooms in which Beauty is awoken, and their probabilities.

I take it to be clear (though see Footnote 5) that, upon awakening in the white room, Beauty should place $1 / 3$ credence in each of the centered worlds WG/Monday, WO/Monday, and BO/Tuesday. Specifically, I take it that both halfers and thirders will agree on this, because in each of the three possible worlds that have not been ruled out, there is only one awakening (out of two) in the white room. Similarly, upon awakening in the black room, Beauty should place $1 / 3$ credence in each of $\mathrm{BG} /$ Monday, $\mathrm{BO} /$ Monday, and $\mathrm{WO} /$ Tuesday. Note the total symmetry between black and white in this example, which will be essential to my argument.

In fact, the WBG variant is arguably isomorphic to Technicolor Beauty Titelbaum, 2008]. In Technicolor Beauty, the setup is the same as in the original variant of the Sleeping Beauty problem, except an additional coin is tossed. If it comes up one way, Beauty sees a red piece of paper on Monday and (if she wakes up on Tuesday at all) a blue piece of paper on Tuesday; otherwise, the order in which she sees the colored pieces of paper is reversed. This Technicolor coin corresponds to the White / Black coin in our WBG variant, and the original Sleeping Beauty coin corresponds to the Grey / Opposite coin. Staying asleep on Tuesday corresponds to the grey room in the WBG variant. I will stick with the WBG variant here, in part because I believe it brings out the symmetry slightly better, but also, and perhaps more importantly, because it ensures that Beauty always awakens twice. This latter property makes it difficult to imagine that any credences other than those of $1 / 3,1 / 3,1 / 3$ described above could reasonably be considered correct. If one agrees that the corresponding credences are also correct for both halfers and thirders in Technicolor Beauty 5 then all

\footnotetext{
${ }^{5} \mathrm{~A}$ standard interpretation of how the halfer assigns credences in general (e.g., Halpern 2006], Meacham 2008], Briggs 2010]) would indeed, in the Technicolor Beauty variant, assign credences of $1 / 3,1 / 3,1 / 3$ in the possible worlds HR, TR, TB upon seeing a red piece of paper (where the first letter indicates the outcome of the original coin toss and the second letter the color seen on Monday), as these are the possible worlds that are not ruled out by the evidence. This, surprisingly, results in a credence of $1 / 3$ in Heads for the halfer. However, Pittard 2015] has objected to this conclusion and suggested that another interpretation of halfing should be
} 
that follows can also be put in terms of that variant. We are now ready to introduce the Dutch book.

\section{A Dutch Book for the WBG Sleeping Beauty Variant}

Beauty will be offered the following bets.

- Bet 1. This bet will be offered once, right before the experiment. It costs 20 and pays out 42 if coin 2 comes up Grey.

- Bet 2. This bet will be offered once each time that Beauty awakens in the white or the black room, but never in the grey room. Thus, it will be offered once overall if coin 2 comes up Grey, but twice overall if coin 2 comes up Opposite. It costs 24 and pays out 33 if coin 2 comes up Opposite.

It should be noted that these bets are legitimate in the sense that the bookie is not exploiting any information that Beauty does not have available to her. I will revisit this point in Subsection 4.3 below.

found that keeps the credence in Heads at $1 / 2$ in Technicolor Beauty. One interpretation of halfing that would achieve this is to treat Beauty's awakening as selected uniformly at random from her awakenings in the experiment in the actual world. Under this interpretation, we would have $P$ (see red|HR) $=1$ (because HR has only one awakening) but $P$ (see red|TR) = $P($ see red $\mid \mathrm{TB})=1 / 2$ (because in each of TR and TB, only one of two awakenings results in seeing red). Hence, by Bayes' rule,

$$
\begin{gathered}
P(\mathrm{HR} \mid \text { see red })=\frac{P(\text { see red } \mid \mathrm{HR}) P(\mathrm{HR})}{P(\text { see red } \mid \mathrm{HR}) P(\mathrm{HR})+P(\text { see red } \mid \mathrm{TR}) P(\mathrm{TR})+P(\text { see red } \mid \mathrm{TB}) P(\mathrm{~TB})} \\
=\frac{1 \cdot(1 / 4)}{1 \cdot(1 / 4)+(1 / 2) \cdot(1 / 4)+(1 / 2) \cdot(1 / 4)}=1 / 2
\end{gathered}
$$

as desired by Pittard. In contrast, if we apply this interpretation of halfing to the WBG variant, we still obtain

$$
\begin{aligned}
P(\mathrm{WG} \mid \text { see white })= & \frac{P(\text { see white } \mid \mathrm{WG}) P(\mathrm{WG})}{P(\text { see white } \mid \mathrm{WG}) P(\mathrm{WG})+P(\text { see white } \mid \mathrm{WO}) P(\mathrm{WO})+P(\text { see white } \mid \mathrm{BO}) P(\mathrm{BO})} \\
& =\frac{(1 / 2) \cdot(1 / 4)}{(1 / 2) \cdot(1 / 4)+(1 / 2) \cdot(1 / 4)+(1 / 2) \cdot(1 / 4)}=1 / 3
\end{aligned}
$$

as desired for the Dutch book presented in this paper. The key difference from Technicolor Beauty is that $P($ see white $\mid \mathrm{WG})=1 / 2$ because there is also an awakening in the grey room. Of course, the standard interpretation of halfing also results in credences of $1 / 3,1 / 3,1 / 3$ in the WBG variant. The point of discussing this other interpretation of halfing here is not to argue for it, but rather merely to show that while interpretations of halfing may disagree about the correct credences in Technicolor Beauty, it is hard to imagine that they would disagree in the WBG variant. One possible approach to finding an interpretation that disagrees is to take Bostrom's approach of classifying awakenings into "reference classes" Bostrom, 2002, 2007] and argue that the awakenings in the white and black rooms belong to the same reference class, but not those in the grey room, thereby eliminating the grey room from the picture in the calculation above. However, it seems hard to justify this classification without reference to the particular details of the bets offered, and it seems difficult to swallow that credences should depend on these details. 


\begin{tabular}{|r|c|c|c|c|}
\hline & WG $(1 / 4)$ & WO $(1 / 4)$ & BO $(1 / 4)$ & BG $(1 / 4)$ \\
\hline Sunday & bet $1: 22$ & bet $1:-20$ & bet 1: -20 & bet $1: 22$ \\
\hline Monday & bet $2:-24$ & bet $2: 9$ & bet 2: 9 & bet 2: -24 \\
\hline Tuesday & no bet & bet 2: 9 & bet 2: 9 & no bet \\
\hline total gain from accepting all bets & -2 & -2 & -2 & -2 \\
\hline
\end{tabular}

Figure 4: The table shows which bet is offered when, as well as the net gain from accepting the bet in the corresponding possible world, for the Dutch book presented in this paper.

First, let us verify that accepting all these bets is sure to result in a loss. If coin 2 comes up Grey, then bet 2 is offered only once, so that Beauty pays $20+24=44$, and receives a payout of 42 from bet 1 - so she runs a loss of 2 . On the other hand, if coin 2 comes up Opposite, then bet 2 is offered twice, so that Beauty pays $20+2 \cdot 24=68$, and receives a payout of $2 \cdot 33=66$ from the two iterations of bet 2 - so again she runs a loss of 2. The table in Figure 4 summarizes the Dutch book.

But who will actually accept these bets? Both causal and evidential decision theorists will accept bet 1 , because before the experiment there is a $50 \%$ chance that coin 2 comes up Grey, so that the expected payout from bet 1 is 21 , which is greater than 20 . Will a causal decision theorist accept bet 2 ? No: given that the room is (say) white, she believes that there is a probability of $2 / 3$ that coin 2 has come up Opposite, so the expected payout of the bet is $(2 / 3) \cdot 33=22$, which is less than the cost of the bet, 24 . So the causal decision theorist is not vulnerable to this Dutch book.

All that remains to show is that the evidential decision theorist will accept bet 2 whenever it is offered to her. Here, then, is the crux of the argument. Suppose the room is white. Then, accepting the bet is strong evidence that she also would also accept the bet in the black room. After all, the situation (including the bets) is entirely symmetric between white and black, so it is hard to see why Beauty would accept the bet in the white room but not in the black room. Similarly, not accepting the bet is strong evidence that she would also not accept it in the black room. Now, her credence is $2 / 3$ that coin 2 has come up Opposite, in which case she either will be, or has been, confronted with the black room. Accepting the bet now (in the white room) leads her to believe that she accepts on both days in this case, which costs 48 and pays off 66, for a gain of 18; not accepting the bet leads her to believe that she does not accept it on either day. On the other hand, her credence is $1 / 3$ that coin 2 has come up Grey, in which case it must now be Monday and no bet will be offered tomorrow. In this case, accepting the bet costs 24 and pays off nothing, for a loss of 24 . Thus, in expectation, the gain from accepting the bet is $(2 / 3) \cdot 18-(1 / 3) \cdot 24=12-8=4>0$. So she will accept the bet in the white room! Of course, by the symmetry between white and black, this means that she will also accept the bet in the black room. Hence, the evidential decision 
theorist falls for the Dutch book.

Some intuition for what makes this Dutch book work is as follows. From the perspective of maximizing expected net gain, clearly bet 1 is a good one to accept, resulting in an ex ante expected net gain of $(2 / 4) \cdot 22-(2 / 4) \cdot 20=1$. This suggests that the evidential decision theorist's mistake is in accepting bet 2 . Always accepting bet 2 results in an ex ante expected net loss of $(2 / 4) \cdot 24-(2 / 4)$. $18=3$. So what makes the evidential decision theorist accept this bet? Suppose she is in a white room. She will reason that if she accepts, then she would also accept in a black room. There are three possible worlds where she is in a black room at some point: $\mathrm{WO}, \mathrm{BO}$, and $\mathrm{BG}$. But BG is ruled out by the evidence of currently being in a white room and therefore does not factor into her current expected payoff calculation. Moreover, this is precisely the one world where accepting the bet in a black room comes at a cost! Therefore, she evaluates the quality of the bet based on a biased selection of the centered worlds in a black room, making the bet look better than it is. The causal decision theorist, on the other hand, ignores bets in black rooms altogether when making a decision in a white room, and thereby avoids being affected by this selection bias.

\section{Discussion}

What has gone wrong for the evidential decision theorist, particularly the evidential decision theorist who is a halfer and is hence supposed to be immune to Dutch books according to Briggs [2010]? In this section, I first discuss the key technical problem with attempting to apply Briggs' proof in the context of the WBG variant. As noted earlier, a key issue is the possibility that knowledge of a decision in one information state affects beliefs about decisions in a different information state. One way around the Dutch book, therefore, is to deny the possibility of beliefs being affected in this way. I continue by arguing that this escape route is unreasonable. I conclude this section by discussing to what extent susceptibility to the Dutch book indicates irrationality.

\subsection{The problem with attempting to apply Briggs' proof}

Why does Briggs' proof of the invulnerability to Dutch books of evidential decision theorists who are halfers not apply here? To appreciate this, it will be helpful to first discuss some essential features of her proof. As noted earlier, it implicitly assumes that the information that Beauty has available to her upon awakening during the experiment is always the same. Briggs uses $N_{W}$ to refer to the number of centers (awakening events within the experiment) in possible (uncentered) world $W$. Suppose Beauty is considering a bet whose net payout (including the initial cost of the bet) is $X_{W}$ in world $W$. If she is an evidential decision theorist, she will reason that if she accepts (rejects) the bet now, then she also accepts (rejects) it on all other occasions. She concludes that 
her net payout is $N_{W} X_{W}$ if she accepts, and 0 if she rejects 6 Of course she does not necessarily know in which possible world she is, so she has to consider the expected value. Letting $C r_{u}$ denote halfer credences, an evidential decision theorist who is a halfer will accept the bet if $\sum_{W} C r_{u}(W) N_{W} X_{W}>0$.

In contrast, a causal decision theorist deciding on the same bet will not let the other $N_{W}-1$ bets that she is offered in world $W$ affect her decision, so that $N_{W} X_{W}$ is replaced by $X_{W}$ in the above. However, if she is a thirder rather than a halfer, then her credence in world $W$ will be proportional to $C r_{u}(W) N_{W}$ rather than $C r_{u}(W)$. Hence, again, she accepts the bet if $\sum_{W} C r_{u}(W) N_{W} X_{W}>0$, the only difference being that the factor $N_{W}$ comes from the credence in this case. Briggs proves that betting in this way ("betting at thirder odds") leaves Beauty immune to Dutch books.

All of this makes sense when Beauty always has the same information upon awakening. When this is not the case, we should first enrich the notation a bit. What is relevant is not the total number of centers $N_{W}$ in a world, but rather the number of centers $N_{W}^{I}$ consistent with the current information $I$. For example, in the WBG variant, it does not suffice to know that $N_{\mathrm{WG}}=2$; rather, we need that $N_{\mathrm{WG}}^{\text {white }}=1, N_{\mathrm{WG}}^{\text {grey }}=1$, and $N_{\mathrm{WG}}^{\text {black }}=0$. Then, one might suppose that with information $I$, the credence in some world $W$ (that is not yet ruled out by $I)$ is $C r_{u}(W)$ for the halfer and proportional to $C r_{u}(W) N_{W}^{I}$ for the thirder. (Note that this would be consistent with the credences in the WBG variant.) The causal decision theorist who is a thirder would then accept the bet if $\sum_{W} C r_{u}(W) N_{W}^{I} X_{W}>0$. Now, what about the evidential decision theorist who is a halfer? Suppose it were the case that now accepting (rejecting) the bet with information $I$ leads her to believe that she always accepts (rejects) it with information $I$, but does not influence her beliefs about what she would do given any other information 7 Then, in world $W$, she believes her net payout if she rejects the bet is $c$ (i.e., whatever she expects to get from any bets accepted when she has information other than $I$ ), and her net payout if she accepts the bet is $N_{W}^{I} X_{W}+c$. The $c$ term cancels out, and hence, again, she will accept the bet if $\sum_{W} C r_{u}(W) N_{W}^{I} X_{W}>0$. So the argument would appear to carry through. The problem is that, as I have argued (and will argue further in Subsection 4.2), it is unreasonable to suppose that the decision made with the current information does not affect beliefs about decisions made with slightly different information! If it does affect them, then the equivalence argument falls apart: we can no longer cancel out the $c$ term in the above because it now depends on the decision made with information $I$, and as a result the condition for accepting a bet changes in the case of the evidential decision theorist who is a halfer. This is what allows the Dutch book for the WBG variant. (It is worth emphasizing again that in the WBG variant, the credences of $1 / 3$ placed in each of the remaining possible worlds do not seem in question, suggesting that the

\footnotetext{
${ }^{6}$ This assumes that she will be offered the same bet upon each awakening, but this is a reasonable requirement: see Subsection 4.3

${ }^{7}$ Again, note that she should always be offered the same bet whenever she has information $I$; otherwise, the bet offered would in fact give her additional information. See Subsection 4.3 for further discussion.
} 
problem in fact lies with evidential decision theory, not with halfing - at least as far as this particular Dutch book is concerned.)

Thus, a causal decision theorist who is a thirder might analyze the evidential decision theorist's susceptibility to the Dutch book as follows. When an evidential decision theorist considers the payoff she expects to get from a given action in some centered world $C$, what happens is that, for each possible world $W$ with multiple centers that are like $C$, she counts the effects of the action multiple times in $W$. This is a mistake. On the other hand, when a halfer in centered world $C$ assesses the probability of a possible world $W$ that contains multiple centers that are indistinguishable from $C$, she fails to give probability to $W$ that is proportional to the number of such centers in $W$. This, too, is a mistake. However, the two mistakes happen to cancel each other out exactly, if the only centers that are like $C$ are the centers that are indistinguishable from $C$. Typical Sleeping Beauty variants have this feature, giving rise to the idea that halfers who adopt evidential decision theory avoid Dutch books. However, it is possible for two centered worlds to be alike while simultaneously being distinguishable. This is what is happening in the WBG variant - white centered worlds and black centered worlds are alike but distinguishable. Because of this, the mistake in assessing actions' payoffs is still made, but it is not canceled out because no mistake is made in assessing the probabilities of possible worlds.

\subsection{Avoiding the Dutch book by not changing beliefs in other information states}

Based on the above, one strategy for the evidential decision theorist to avoid the Dutch book is to never let decisions in one information state affect beliefs about decisions in different information states. Suppose she takes this approach and we vary the decision that she makes in the current centered world. As we do so, her beliefs (conditional on this decision) about what she would do in centered worlds that are indistinguishable from the current one will also vary, but her beliefs about what she would do in other centered worlds (in particular, ones that are alike but distinguishable) will not. This indeed avoids the Dutch book. But this approach seems highly unappealing.

Of course, one could add details to the case to make this approach seem more palatable. For example, we may suppose that before the experiment, a neurological examination revealed to Beauty that the part of her brain that is activated to make decisions in white rooms is entirely disjoint from the part activated to make decisions in black rooms. With these (or perhaps alternative) additional details, it can perhaps be successfully argued that her beliefs about what she does in black rooms should not be affected by information about what she does in white rooms. But this fails to get the evidential decision theorist out of trouble. The Dutch book does not need to succeed no matter what details are added to the case. For it to exhibit a problem with evidential decision theory, all that is necessary is that is succeeds for some details. We may just as well specify that the neurological examination reveals to Beauty that the part of her brain involved in decision making is entirely uninfluenced by the color of the 
room. In that case, it seems entirely unreasonable for beliefs about black-room decisions to be uninfluenced by knowledge of white-room decisions.

Moreover, even though this is in fact not necessary for the argument to succeed, I would argue that when no details are added to the case - i.e., Beauty does not have any additional relevant information, such as the results of a neurological examination - by default, beliefs about black-room decisions should be influenced by knowledge of white-room decisions as I have suggested. By way of analogy, suppose we see Kim treating another person kindly, and this other person happens to stand to her left. Clearly, this will increase our credence that Kim would treat other people who stand to her left kindly. But it would be preposterous to not also increase our credence that Kim would treat people who stand to her right kindly, unless we have reason to believe that there is a fundamental asymmetry between left and right (e.g., if we know Kim does not hear well with her right ear and this causes her great frustration). The situation is similar in our context: unless we have a particular reason to believe that the color of the room is relevant to the decisions (as in the first example of a neurological examination), the Dutch book goes through 8

\subsection{Why susceptibility to (certain) Dutch books poses a problem}

Does susceptibility to diachronic Dutch books really indicate irrationality? This question has been discussed at length in earlier work Hitchcock, 2004, Briggs, 2010], and I do not have much to add that is new, but it is worth revisiting the key points here. Some Dutch book arguments have been made that require the bookie to have information that Beauty does not. For example, consider the following Dutch book argument against a thirder, presented by Hitchcock 2004] precisely in order to highlight this issue. On Sunday, Beauty is offered a bet that pays out 30 on Heads, which costs 15 . Then, on Monday, Beauty is offered a bet that pays out 30 on Tails, which costs 20 . The argument is that she is willing to accept both bets - in particular, she is willing to accept the latter because she at that point believes the probability of Tails is $2 / 3$ - but accepting both bets is sure to result in a loss of 5 . The problem is that offering the second bet requires the bookie to know something that Beauty does not, namely, that it is Monday and not Tuesday. If he did not know this, he could end up selling the second bet to her twice in the Tails world, which would cause the Dutch book to fail 9 Now, susceptibility to being Dutch-booked by a bookie that has additional information does not seem to indicate a failure of rationality. After all, consider

\footnotetext{
${ }^{8}$ One might also suppose that knowledge of her decision in a white room makes Beauty only (say) $99 \%$ confident in what her decision would be in a black room, where the remaining $1 \%$ is intended to capture a small probability that the room color is somehow relevant to the decision. It is easy to see that the Dutch book still goes through under these conditions.

${ }^{9}$ As already pointed out by Hitchcock, to be precise, what information the bookie has is not exactly what is at issue. If the bookie does not know what day it is, but someone else prevents the bookie from offering the second bet on Tuesday to make the Dutch book work, this is just as problematic. The point is that the process as a whole by which bets are offered to Beauty cannot use information that is unavailable to Beauty.
} 


\begin{tabular}{|c|c|c|c|}
\hline & W $(1 / 4)$ & WB $(1 / 2)$ & B (1/4) \\
\hline Monday & White awakens & both awaken & Black awakens \\
\hline
\end{tabular}

Figure 5: Possible worlds and their probabilities with two Beauties.

the extreme case where a deceptive bookie already knows the outcomes of the bets in advance. In such a case it is not surprising that he can choose which bet to offer to ensure himself a profit. (For a concrete example, see Briggs [2010].) Another take on this is that if Beauty is astute, then the event of being offered a bet in fact provides her with additional information, which she should take into account when deciding whether to accept. If our thirder Beauty above knows that the second bet is only offered to her on Mondays, then upon being offered the bet she will know it is Monday, update her credence in Tails to $1 / 2$, and not take the bet.

However, like Hitchcock's Dutch book, the Dutch book presented in this paper does not require the bookie to have superior information. It is sufficient for the bookie to know what Beauty knows (i.e., the color of the room) in order to decide which bet to offer her. In Hitchcock's words, he can "sleep with her" that is, be put to sleep and awoken and have his memory impaired in exactly the same manner. As a result, being offered a bet can never provide Beauty with additional information. This remains true even if she is told the bookie's entire betting strategy at the outset. This is perhaps what most strongly suggests that susceptibility to such a Dutch book indicates a degree of irrationality: even if Beauty is completely aware of the game the bookie is playing with her, she still falls for the sure loss.

\section{Concluding Remarks}

The evidential decision theorist may hope that the type of Dutch book presented here is inherently restricted to scenarios where the agent's memory is impaired. But I believe that the problem runs at least a bit deeper than that. For example, we can easily modify the WBG variant so that there are now two Beauties, one ("White") who is awoken whenever the original Beauty was awoken in the white room, and one ("Black") who is awoken whenever the original Beauty was awoken in the black room. These Beauties can be awoken simultaneously (in separate rooms) rather than sequentially, thereby combining the WO and $\mathrm{BO}$ worlds into a single WB world. The table in Figure 5 summarizes this variant. We can then let the two Beauties bet under a joint account whose value they are both trying to maximize, and, if they are evidential decision theorists, they will fall prey to the same Dutch book, even without memory impairment (assuming no communication between them). The table in Figure 6 summarizes the Dutch book. For the purpose of symmetry, we split bet 1 into two halves, each denoted 1', one for White and one for Black, with half the cost and half the payout each. Note that in this context, my interpretation 


\begin{tabular}{|r|c|c|c|}
\hline & W $(1 / 4)$ & WB $(1 / 2)$ & B $(1 / 4)$ \\
\hline Sunday & $\begin{array}{c}\text { bet 1' }(\mathrm{W}): 11 \\
\text { bet 1' }(\mathrm{B}): 11\end{array}$ & $\begin{array}{c}\text { bet 1' }(\mathrm{W}):-10 \\
\text { bet 1' }(\mathrm{B}):-10\end{array}$ & $\begin{array}{c}\text { bet 1' }(\mathrm{W}): 11 \\
\text { bet 1' (B): 11 }\end{array}$ \\
\hline Monday & $\begin{array}{c}\text { bet 2 (W): }-24 \\
\text { (no bet for B) }\end{array}$ & $\begin{array}{c}\text { bet 2 (W): } \\
\text { bet 2 }(\mathrm{B}): 9\end{array}$ & $\begin{array}{c}\text { (no bet for W) } \\
\text { bet 2 (B): }-24\end{array}$ \\
\hline total gain from accepting all bets & -2 & -2 & -2 \\
\hline
\end{tabular}

Figure 6: The table shows which bet is offered when and (in parentheses) to whom, as well as the net gain from accepting the bet in the corresponding possible world, for the Dutch book adapted to the two-Beauties variant.

of evidential decision theory comes down to requiring that each Beauty, when calculating her expected utility from accepting a bet (or declining it), assumes in this calculation that the other Beauty would do the same - so we should assume that the Beauties are psychological twins 10 Specifically, when offered bet 2 , White places credence $2 / 3$ in the world $\mathrm{WB}$, and when calculating the value of accepting bet 2 in this world assumes that Black will accept too, for a total gain of $2 \cdot 9=18$; she places credence $1 / 3$ in the world $W$, where Black will not get to act, so that the loss from accepting the bet is only 24 . Because (once again) $(2 / 3) \cdot 18-(1 / 3) \cdot 24=12-8=4>0$, she accepts the bet (and Black will do so as well, by symmetry).

Does the fact that the two Beauties together are susceptible to a Dutch book indicate that they are irrational? This is certainly not as well established as it is for the case of a Dutch book for a single agent (as summarized in Subsection 4.3), and some skepticism is in order. For example, it is well known in game theory that rational behavior by multiple agents can result in an outcome that is strongly Pareto dominated, i.e., there exists another outcome that all agents would strictly prefer. The Prisoner's Dilemma is the standard example. However, such examples rely on the agents having different preferences. In contrast, the two Beauties above have the exact same preferences. Also, it seems that the key properties that make a Dutch book convincing, as discussed in Subsection 4.3. still hold here. It is true that the bookie will have more information than either single Beauty alone. However, this is easily fixed by stipulating that there are two bookies, also with a joint account, each of whom is assigned to sleep with and offer bets to one of the Beauties. Then, again, being offered a bet does not provide either Beauty with more information, and this remains true even if the bookies' joint betting strategy is common knowledge at the outset.

It appears, then, that the Dutch book argument presented in this paper deals a serious blow to evidential decision theory. Of course, evidential decision theory is often applied in settings where a decision provides evidence not about past or future decisions that are similar, or about decisions by another similar

\footnotetext{
${ }^{10}$ Variants of the Sleeping Beauty problem with clones are fairly common - see, e.g., Elga 2004] and Schwarz 2014a].
} 
agent, but rather about something relevant in the environment - various types of brain lesion, a demon's decision, etc. It does not appear that this Dutch book argument can be applied in such cases, so perhaps the evidential decision theorist can retreat to an appropriately restricted version of the theory (though it is not immediately clear whether and how this can be coherently done). Even upon such retreat, I believe the glancing blow to what remains of the theory should be cause for concern for anyone sympathetic to it.

\section{Acknowledgments}

I thank the anonymous reviewers for many useful comments that have helped to significantly improve the paper.

\section{References}

Frank Arntzenius. Reflections on Sleeping Beauty. Analysis, 62(1):53-62, 2002.

Nick Bostrom. Anthropic Bias: Observation Selection Effects in Science and Philosophy. Routledge, 2002.

Nick Bostrom. Sleeping Beauty and Self-location: A Hybrid Model. Synthese, 157(1):59-78, 2007.

Rachael Briggs. Putting a value on Beauty. In Tamar Szabó Gendler and John Hawthorne, editors, Oxford Studies in Epistemology: Volume 3, pages 3-34. Oxford University Press, 2010.

Kai Draper and Joel Pust. Diachronic Dutch Books and Sleeping Beauty. Synthese, 164(2):281-287, 2008.

Adam Elga. Self-locating belief and the Sleeping Beauty problem. Analysis, 60 (2):143-147, 2000 .

Adam Elga. Defeating Dr. Evil with self-locating belief. Philosophy and Phenomenological Research, 69(2):383-396, 2004.

Joseph Y. Halpern. Sleeping Beauty reconsidered: Conditioning and reflection in asynchronous systems. In Tamar Szabó Gendler and John Hawthorne, editors, Oxford Studies in Epistemology: Volume 3, pages 111-142. Oxford University Press, 2006.

Christopher Hitchcock. Beauty and the bets. Synthese, 139(3):405-420, 2004.

Christopher J. G. Meacham. Sleeping beauty and the dynamics of de se beliefs. Philosophical Studies, 138(2):245-269, 2008. 
John Pittard. When Beauties disagree: Why halfers should affirm robust perspectivalim. In Tamar Szabó Gendler and John Hawthorne, editors, Oxford Studies in Epistemology: Volume 5. Oxford University Press, 2015. Forthcoming.

Wolfgang Schwarz. Belief update across fission. British Journal for the Philosophy of Science, 2014a. Forthcoming.

Wolfgang Schwarz. Lost memories and useless coins: Revisiting the absentminded driver, 2014b.

Michael G. Titelbaum. The relevance of self-locating beliefs. Philosophical Review, 117(4):555-605, 2008.

Michael G. Titelbaum. Ten reasons to care about the Sleeping Beauty problem. Philosophy Compass, 8(11):1003-1017, 2013. 